\title{
Assessment of hand hygiene practice among residents of Uyo-Southern Nigeria during the COVID-19 pandemic.
}

\author{
Ubani, Blessing Chinenye ${ }^{1,2}$, Ekuma, Agantem Emmanuel ${ }^{2,3}$, \\ Akpabio, Akpabio Akanimo ${ }^{1}$, Akpabio, Tiemah Ntiense ${ }^{1}$ \\ ${ }^{1}$ Department of Internal Medicine, University of Uyo Teaching Hospital, Uyo, Akwa Ibom State, Nigeria \\ 2University of Uyo, Uyo, Akwa Ibom State, Nigeria \\ ${ }^{3}$ Department of Clinical Microbiology, University of Uyo Teaching Hospital, Uyo, Akwa Ibom State, Nigeria
}

DOI: 10.3396/ijic.v16i3.017.20

\begin{abstract}
The COVID19 pandemic has caused widespread global devastation with over 11 million infections and 534,000 deaths recorded at the end of June 2020. Measures effective in controlling the disease include physical distancing and regular hand hygiene, though compliance varies. This study aimed to determine the frequency of hand hygiene compliance, identify reasons for non-compliance as well as significant associations of hand hygiene performance, type and duration. Visitors to two landmark sites in Uyo, southern Nigeria (a tertiary hospital and a popular mall) were observed for hand hygiene compliance. The type and duration of hand hygiene was also assessed. A subset of the participants was subsequently invited to participate in an interviewer-administered survey comprising questions on hand hygiene frequency, type, duration and reasons for non-compliance. Ethical approval was obtained for the study. A total of 490 subjects were observed from both sites ( 280 from the mall and 210 from the hospital), while 155 , comprising $52.9 \%$ females and $47.1 \%$ males, with a mean age of $32.3 \pm 11.2$ years completed the survey. Although almost all participants (94.2\%) considered hand hygiene a necessity, most (65.2\%) forgot to wash their hands. The majority of the participants (74.3\%) performed hand hygiene, and using soap and running water was the preferred method (80.6\%). Age and location were significantly associated with hand hygiene performance, type and duration.
\end{abstract}

Over $70 \%$ of adult participants in Uyo performed hand hygiene, though infrequently. Forgetfulness was the most common reason for non-compliance. Public education and compulsion at public places are key strategies in improving hand hygiene compliance.

Keywords: hand hygiene, observation, coronavirus infection, Nigeria.

Corresponding author:

Ubani Blessing Chinenye

Department of Internal Medicine,

University of Uyo Teaching Hospital, Uyo - Nigeria

Email: blessiykebani@gmail.com 


\section{Background}

Since its discovery in Wuhan China in December, 2019, the novel coronavirus infection named COVID-19, has spread to over 227 countries globally, with more than 11 million people infected and over 528,000 deaths recorded, as of $5^{\text {th }}$ July 2020.

The disease was declared a global pandemic by the World Health Organization (WHO), in less than three months following its outbreak. ${ }^{2}$ The pandemic quickly spread to North Africa from Europe by mid-February 2020 and about two weeks later Nigeria recorded the first case in sub-Saharan Africa. Currently, Nigeria has over 28, 167 cases with over 634 deaths. ${ }^{3}$ Following initial cases in large cities such as Lagos and Abuja, the disease spread to different parts of the country. There is no cure or therapeutic vaccine against the virus at the moment, hence preventive measures are the current approach to reduce its spread. The WHO has recommended effective measures to prevent or reduce disease transmission which include physical distancing, respiratory hygiene and regular hand hygiene. ${ }^{4}$ Hand hygiene is a very important measure for the prevention and control of COVID-19 as well as other viruses and bacteria that cause common colds, flu and pneumonia. The WHO recommends direct observation as the standard for monitoring hand hygiene compliance. Direct observation of hand hygiene can be affected by the Hawthorne effect as well as inter-observer variation. ${ }^{5}$ Hand hygiene compliance ranges widely from $5 \%$ to $89 \%$, with an average of $38.7 \%$ among health care workers. ${ }^{6}$ Poor hand washing practices have also been observed among non-health workers in Nigeria, even where there is constant availability of soap and water for such people. ${ }^{7}$

Despite a high awareness of the importance of hand hygiene in preventing viral and bacterial infections, access to hand hygiene facilities which include alcohol-based hand sanitizers as well as soap and water is often inadequate in the communities and health care outlets, with developing countries being the worst affected. According to WHO and UNICEF an estimated 3 billion people worldwide lack hand hygiene facilities at home and two out of five health care facilities lack hand hygiene at points of care. ${ }^{8}$ The $\mathrm{WHO}$ has recommended the provision of hand hygiene facilities like running water, soap and sanitizers at the entrance of all public places to ensure compliance with the WASH (Wash Sanitize Hygiene) strategy as a way of curbing the spread of COVID-19. ${ }^{4}$ This study is aimed at observing hand hygiene compliance among adult residents of Uyo, Akwa Ibom State - Nigeria, as well as determining the reasons for failure to observe hand hygiene in the era of the COVID-19 pandemic. This will help in identifying areas that need improvement.

\section{Methods}

\section{Setting}

This study was carried out in Uyo, the capital city of Akwa Ibom State, South-South Nigeria. This was one of the states that recorded early cases of COVID-19 in Nigeria. A total lock down of movement was instituted in the state for a period of four weeks after which movement was allowed with requirements for social distancing, compulsory use of facemasks and hand hygiene at public places instituted. Two locations were used for this study; a 500-bed government-owned tertiary hospital with over 400 patient visits per day and roughly 2,000 staff, and a large privately owned shopping mall located at the city center popular among city residents, containing a supermarket, restaurant, and pharmacy. Both sites have conspicuously located hand washing points and security personnel to ensure compliance.

\section{Hand hygiene observation}

We observed the hand hygiene compliance of every second visitor at each site for a total duration of two hours. The visitors were unaware of the presence of the observer in order to avoid a Hawthorne effect. Each subject was observed for hand hygiene performance (done without compulsion, done with compulsion, not done), type of hand hygiene (soap and water, or alcohol-based sanitizer), and duration of hand hygiene ( $>20$ seconds, and $<20$ seconds). The observer also recorded their gender and estimated age range (young, middle-aged or elderly).

\section{Hand hygiene attitude and practice}

In order to ascertain their attitude towards hand washing, we invited a subset of our subjects to participate in a survey using an intervieweradministered questionnaire. Every fourth visitor 
Table I. Socio-demographic characteristics and hand hygiene performance of participants in the observation study

Observation study

\begin{tabular}{llrrr}
\hline \multirow{2}{*}{$\mathbf{n}(\%)$} & & Hospital $(\mathrm{n}=210)$ & Mall (n=280) & Total $(\mathrm{n}=490)$ \\
\cline { 3 - 5 } & & $\mathrm{n}(\%)$ & $\mathrm{n}(\%)$ \\
\hline \multirow{2}{*}{ Age } & Young & $101(48.1)$ & $148(52.9)$ & $249(50.8)$ \\
& Middle-aged & $83(39.5)$ & $114(40.7)$ & $197(40.2)$ \\
& Elderly & $26(12.4)$ & $18(6.4)$ & $44(9.0)$ \\
\multirow{2}{*}{ Gender } & Male & $97(46.2)$ & $145(51.8)$ & $242(49.4)$ \\
& Female & $113(53.8)$ & $135(48.2)$ & $248(50.6)$ \\
\hline \multirow{2}{*}{ Hand Hygiene } & Compulsion & $0(0)^{*}$ & $22(7.9)$ & $22(4.5)$ \\
performed & No-compulsion & $84(40.0)$ & $258(92.1)$ & $342(69.8)$ \\
& Not done & $126(60.0)$ & $0(0)^{*}$ & $126(25.7)$ \\
\hline
\end{tabular}

*Hand hygiene was compulsory for entry into the mall but not at the hospital.

Table II. Socio-demographic characteristics of participants in the survey study

\begin{tabular}{|c|c|c|c|c|}
\hline \multicolumn{5}{|c|}{ Survey } \\
\hline \multirow{2}{*}{$n(\%)$} & & Hospital (n=78) & Mall $(n=77)$ & Total $(n=155)$ \\
\hline & & $\mathrm{n}(\%)$ & $n(\%)$ & \\
\hline Age & $\pm \mathrm{SD}$ (mean) & $\begin{array}{r}31.69 \pm 11.32 \\
(29.00)\end{array}$ & $\begin{array}{r}33.01 \pm 11.08 \\
(30.00)\end{array}$ & $\begin{array}{r}32.35 \pm 11.19 \\
(30.00)\end{array}$ \\
\hline \multirow{2}{*}{ Sex } & Male & $38(48.7)$ & $35(45.5)$ & $73(47.1)$ \\
\hline & Female & $40(51.3)$ & $42(54.5)$ & $82(52.9)$ \\
\hline \multirow{4}{*}{ Marital status } & Single & 49 (62.8) & $46(59.7)$ & 95 (61.3) \\
\hline & Married & $24(30.8)$ & 27 (35.1) & $51(32.9)$ \\
\hline & Divorced & $4(5.1)$ & $2(2.6)$ & $6(3.9)$ \\
\hline & Separated & $1(1.3 \%)$ & $2(2.6)$ & $3(1.9)$ \\
\hline \multirow{4}{*}{ Education } & Informal & $2(2.6)$ & $1(1.3)$ & $3(1.9)$ \\
\hline & Primary & $0(0)$ & $5(6.5)$ & $5(3.2)$ \\
\hline & Secondary & $20(25.6)$ & $25(32.5)$ & $45(29.0)$ \\
\hline & Tertiary & $56(71.8)$ & $46(59.7)$ & 102 (65.8) \\
\hline \multirow{4}{*}{ Occupation } & Unemployed & $17(21.8)$ & $13(16.9)$ & $30(19.4)$ \\
\hline & Public servant & 25 (32.1) & $28(36.4)$ & $53(34.2)$ \\
\hline & Pensioner & $34(43.6)$ & $34(44.2)$ & 68 (43.9) \\
\hline & Business & $2(2.6)$ & $2(2.6)$ & $4(2.6)$ \\
\hline
\end{tabular}


was approached and invited to participate. After giving consent, they were assessed using a short questionnaire, comprising sociodemographic data and questions on reasons for hand hygiene, frequency of hand hygiene, duration of hand hygiene, as well as type and preferred method of hand hygiene.

\section{Data analysis}

Appropriate statistical tests were used for qualitative and quantitative data with $p$ value $<0.05$ considered significant. Ethical approval was obtained for the study.

\section{Ethical approval}

This study was approved by the University of Uyo Teaching Hospital Health Research Ethics Committee.

\section{Results:}

A total of 490 subjects were observed from both sites (280 from the mall, 210 from the hospital) with their gender and estimated age distribution shown in
Table I. From these, 155 participants (52.9\% females, $47.1 \%$ males) with a mean age of $32.3 \pm 11.2$ years completed the survey as shown in Table II. At both sites, participants were mostly single, pensioners with tertiary level of education. Other socio-demographic characteristics of the surveyed participants are shown in Table II.

Regarding hand hygiene practice, although most participants from both sites washed their hands three to five times daily, more people from the hospital (37.2 vs $24.7 \%$ ) washed their hands over 10 times daily as shown in Table III. A third of the surveyed participants performed hand hygiene for $>20$ seconds with nearly an equal number (29.7\%) washing for just 5-10 seconds. Although almost all patients (94.2\%) considered hand hygiene a necessity, most (65.2\%) forgot to wash their hands. Washing with soap and running water was the preferred method by the majority at both sites (80.6\%) with only a minority (19.6\%) having a preference for hand sanitizers.

Table III. Self-reported hand hygiene practice of participants in the survey

\begin{tabular}{llrrr}
$\mathbf{n}$ (\%) & & Hospital (n=78) & Mall (n=77) & Total (n=155) \\
& & $\mathbf{n}(\%)$ & $\mathbf{n}(\%)$ & \\
\hline Frequency of hand & $<2$ times & $4(5.1)$ & $8(10.4)$ & $12(7.7)$ \\
hygiene & 3-5 times & $30(38.5)$ & $33(42.9)$ & $63(40.6)$ \\
& 6-10 times & $15(19.2)$ & $17(22.1)$ & $32(20.6)$ \\
& $>10$ times & $29(37.2)$ & $19(24.7)$ & $48(31.0)$ \\
\hline Duration of hand & 5 sec & $12(15.4)$ & $16(20.8)$ & $28(18.1)$ \\
hygiene & $5-10$ sec & $21(26.9)$ & $25(32.5)$ & $46(29.7)$ \\
& $11-20$ sec & $18(23.1)$ & $12(15.6)$ & $30(19.4)$ \\
& $>20$ sec & $27(34.6)$ & $24(31.2)$ & $51(32.9)$ \\
\hline Reasons why hand & I forget to do so & $52(66.7)$ & $49(63.6)$ & $101(65.2)$ \\
hygiene is not & It affects my hands & $6(7.7)$ & $7(9.1)$ & $13(8.4)$ \\
performed & I do not consider it necessary & $2(2.6)$ & $6(7.8)$ & $8(5.2)$ \\
& My hands do not look dirty & $6(7.7)$ & $5(6.5)$ & $11(7.1)$ \\
& No water to wash hands & $12(15.4)$ & $10(13.0)$ & $22(14.2)$ \\
\hline Do you consider & Yes & $76(97.4)$ & $70(90.9)$ & $146(94.2)$ \\
regular hand hygiene & No & $1(1.3)$ & $5(6.5)$ & $6(3.9)$ \\
a necessity & I don't know & $1(1.3)$ & $2(2.6)$ & $3(1.9)$ \\
\hline Preferred hand & Soap and running water & $63(80.8)$ & $62(80.5)$ & $125(80.6)$ \\
hygiene method & Alcohol-based hand sanitizer & $15(19.2)$ & $15(19.5)$ & $30(19.4)$ \\
\hline
\end{tabular}


Table IV. Factors associated with observed hand hygiene practice

\begin{tabular}{llccc} 
Variable & & \multicolumn{2}{c}{ Hand hygiene performed } & P \\
\cline { 3 - 4 } & & Yes & No & \\
\hline Age & Young & 192 & 57 & 0.018 \\
& Middle-aged & 147 & 50 & \\
& Elderly & 25 & 19 & \\
\hline \multirow{2}{*}{ Gender } & Male & 184 & 58 & 0.382 \\
& Female & 180 & 68 & \\
\hline \multirow{2}{*}{ Location } & Hospital & 84 & 126 & ${ }^{*} 2 \times 10^{\wedge} 5$ \\
& Mall & 280 & 0 & \\
\hline
\end{tabular}

Variable

Type of hand hygiene

p

\begin{tabular}{llccccc}
\cline { 3 - 6 } Age & Washing & sanitizing & None & Both & \\
\hline & Young & 40 & 60 & 82 & 82 & 0.001 \\
& Middle-aged & 47 & 48 & 75 & 75 & \\
& Elderly & 8 & 20 & 11 & 11 & \\
\hline \multirow{2}{*}{ Gender } & Male & 48 & 59 & 83 & 83 & 0.831 \\
& Female & 47 & 69 & 85 & 85 & \\
\hline \multirow{2}{*}{ Location } & Hospital & 45 & 128 & 0 & 0 & ${ }^{*} 2 \times 10^{\wedge 5}$ \\
& Mall & 50 & 0 & 168 & 168 & \\
\hline
\end{tabular}

Variable

Duration of hand hygiene

p

\begin{tabular}{llcccc}
\cline { 3 - 5 } & & $>20 \mathrm{~s}$ & $<20 \mathrm{~s}$ & None & \\
\hline \multirow{2}{*}{ Age } & Young & 152 & 41 & 56 & 0.022 \\
& Middle-aged & 105 & 39 & 53 & \\
& Elderly & 16 & 9 & 19 & \\
\hline \multirow{2}{*}{ Gender } & Male & 142 & 42 & 58 & 0.411 \\
& Female & 131 & 47 & 70 & \\
\hline \multirow{2}{*}{ Location } & Hospital & 48 & 34 & 128 & $* 2 \times 10^{\wedge}$ \\
& Mall & 225 & 55 & 0 & \\
\hline
\end{tabular}

From Table IV, a total of 364 out of 490 participants performed hand hygiene at both sites yielding a frequency of $74.3 \%$. In addition, observation data demonstrate that hand hygiene was done with compulsion in 22 (4.5\%) of participants - all at the mall. Further analyses identified age and location as factors significantly associated with hand hygiene performance as well as type and duration of hand hygiene as shown in Table IV.
Discussion

Our survey participants consisted of young adults, spread equally between both study sites. There was a similar age and gender distribution among the observed participants. In this study, the highest percentage of respondents reported performing hand hygiene three to five times daily. In a similar population hand hygiene study from Sweden prior to the pandemic, higher frequencies of five to nine times daily were reported. ${ }^{9}$ Compared to the Swedes, 
our study participants may be washing their hands infrequently considering the average daily activities of an active young population.

The majority of participants reported hand washing longer than 20 seconds. This was corroborated by direct observation which found an even higher proportion of persons spending longer than 20 seconds on hand hygiene. Duration of hand hygiene has been shown comprehensively to affect the effectiveness of removal of pathogens with up to 30 seconds of hand washing required to completely remove pathogens from both hands. ${ }^{10}$

The most common reason for not washing hands was given as forgetfulness. In a similar observational study from Ghana, $82.2 \%$ and $97.8 \%$ of bus stations had no hand hygiene posters or audio announcements respectively. ${ }^{11}$ This highlights the importance of information, education and communication materials and other reminders in ensuring adherence to hand hygiene guidelines. Reminders in the workplace are a key component of the WHO multimodal hand hygiene improvement strategy. ${ }^{12-14}$ A compulsory hand washing policy prior to entry by many supermarkets has improved hand washing rates as demonstrated in this study.

Most participants selected hand washing with soap and water as their preferred method of hand hygiene over use of alcohol-based hand sanitizers. This response may be partly attributable to current hygiene campaigns which have emphasized washing with soap and water with alcohol sanitizers as an alternative. It is also possible that some hand sanitizers have an unpleasant odour which might put off some potential users. Most healthcare based studies however have shown a clear superiority of alcohol based solution in removing pathogens after hand hygiene compared to using soap and water ${ }^{15}$ except in the case of visibly soiled hands. ${ }^{16}$

We noticed a very widespread use of both hand washing and alcohol hand rub by a large proportion of participants. This practice was mainly carried out in the private facilities where observations were made. This practice has not been reported previously to our knowledge and it is not known what effect it could have on removal of pathogens although it is doubtful that it will provide any additional protection. Frequent and repeated use of hand hygiene products, particularly soaps and other detergents, is an important cause of chronic irritant contact dermatitis. ${ }^{17}$

Skin that is damaged by repeated exposure to detergents may be more susceptible to irritation by all types of hand antiseptic formulations, including alcohol-based preparations. ${ }^{18}$ Another potential danger of this practice is the rapid depletion of hand hygiene materials in a resource-limited setting which could possibly deprive others from the use of these materials later on. There should be sufficient guidance to visitors on proper selection of hand hygiene method followed by the application of hypoallergenic balm. The most important factor associated with hand hygiene was the location. This suggests that institutional factors, probably like the location of hand hygiene facilities and restriction of entry to non-compliers, were the most important drivers for compliance of visitors.

Public places with unrestricted entry and nonenforcement may show lower rates of hand hygiene compliance as reported in Ghana where hand hygiene facilities were infrequently used at $87.4 \%$ of bus stations. ${ }^{11}$ This was also the case from our observation cohort where $4.5 \%$ of participants were compelled to wash their hands, all at the mall. Hand hygiene was compulsory before entry at the mall but was optional at the hospital gate. Although the age range of visitors appeared to be associated with hand hygiene compliance in our study, this association is probably due to the difference in age of persons observed in different locations with more elderly persons observed in the public hospital.

The WHO multimodal hand hygiene improvement strategy has emphasized the importance of institutional preparedness, including allocation of resources, provision of infrastructure, planning and leadership in improving hand hygiene in healthcare settings. ${ }^{12}$ Our findings suggest that similar institutional measures are required to encourage hand hygiene at the population level in efforts to curtail the impact of the COVID-19 pandemic. Some limitations of this study include its observational nature which restricts interpretation of any identified associations, possible selection bias arising from the chosen study 
sites, and scarce African literature on hand hygiene in community settings.

In conclusion, only $74.3 \%$ of adult residents in UyoNigeria performed hand hygiene mostly infrequently (three to five times/day) with few (4.5\%) requiring compulsion. Forgetfulness was the most frequent reason for non-compliance. Intense public education on hand hygiene and compulsory hand washing before entry at public places will be key strategies in improving compliance and reducing community spread.

\section{Ethics}

Approval obtained for the study

\section{Conflict of interest}

The authors have no conflict of interest to declare

\section{Funding}

None

\section{Acknowledgements}

The authors wish to thank Drs. Ememe Michael, Hilary Olise, Enwongo Ekanem and Simeon Jack for their assistance with data collection.

\section{References}

1. World Health Organization Coronavirus Disease (COVID-19) Dashboard | WHO Coronavirus Disease (COVID-19) Dashboard, (accessed 5 July 2020).

2. Cucinotta D, Vanelli M. WHO declares COVID-19 a pandemic. Acta bio-medica: Atenei Parmensis 2020; 91: 157-160.

3. NCDC Coronavirus COVID-19 Microsite, https://covid19. ncdc.gov.ng/ (accessed 5 July 2020).

4. World Health Organization. WASH and COVID-19. Available from: http://www.who.int/water_sanitation_health/newsevents/wash-and-covid-19/en/ (accessed 5 July 2020)

5. Garcell HG, Arias AV, Miranda FR, et al. Direct observation of hand hygiene can show differences in staff compliance: Do we need to evaluate the accuracy for patient safety? Qatar Med J 2017; 1. https://doi.org/10.5339/qmj.2017.1

6. Powell-Jackson T, King JJ, Makungu C, et al. Infection prevention and control compliance in Tanzanian outpatient facilities: a cross-sectional study with implications for the control of COVID-19. Lancet Glob Health, 2020. https://doi. org/10.1016/S2214-109X(20)30222-9
7. Ekanem A, Johnson O. Hand washing practices at critical times among mothers in selected health facilities in Akwa Ibom state. Ibom Med J 2015; 8(1): 1.

8. World Health Organization. Recommendations to Member States to improve hand hygiene practices by providing universal access to public hand hygiene stations to help prevent the transmission of the COVID-19 virus: interim guidance, 1 April 2020.

9. Merk H, Kühlmann-Berenzon S, Linde A, et al. Associations of hand-washing frequency with incidence of acute respiratory tract infection and influenza-like illness in adults: a population-based study in Sweden. BMC Infect Dis 2014; 14: 509. https://doi.org/10.1186/1471-2334-14-509

10. Noskin GA, Stosor V, Cooper I, et al. Recovery of VancomycinResistant Enterococci on Fingertips and Environmental Surfaces. Infect Control Hosp Epidemiol 1995; 16: 577-581. https://doi.org/10.1086/647011

11. Bonful HA, Addo-lartey A, Aheto JMK, et al. Prevention of COVID-19 in Ghana: Compliance audit of selected transportation stations in the Greater Accra region of Ghana. Preprint (Medxriv 2020). https://doi. org/10.1101/2020.06.03.20120196

12. World Health Organization. A Guide to the Implementation of the WHO Multimodal Hand Hygiene Improvement Strategy. (No. WHO/IER/PSP/2009/02). 2009.

13. Schmitz K, Kempker RR, Tenna A, et al. Effectiveness of a multimodal hand hygiene campaign and obstacles to success in Addis. Antimicrob Resist Infect Control 2014; 3(8). https:// doi.org/10.1186/2047-2994-3-8

14. Moro ML, Morsillo F, Nascetti S, et al. Determinants of success and sustainability of the WHO multimodal hand hygiene promotion campaign, Italy, 2007- 2008 and 2014. Euro Surveillance 2017; 22: 30546. https://doi.org/10.2807/15607917.ES.2017.22.23.30546

15. Trick WE, Vernon MO, Hayes RA, et al. Impact of ring wearing on hand contamination and comparison of hand hygiene agents in a hospital. Clin Infect Dis 2003; 36: 1383-1390. https://doi.org/10.1086/374852

16. Larson E. A causal link between handwashing and risk of infection? Examination of the evidence. Infect Control Hosp Epidemiol 1988; 9: 28-36. https://doi.org/10.1086/645729

17. Tupker RA. Detergent and cleansers. In: Van der Valk P, Maibach $\mathrm{H}$ (eds) The irritant contact dermatitis syndrome. New York, NY: CRC Press, 1996, 71-76.

18. Lübbe J, Ruffieux C, van Melle G, et al. Irritancy of the skin disinfectant n-propanol. Contact Dermatitis 2001; 45: 226231. https://doi.org/10.1034/j.1600-0536.2001.450407.x

19. Cavanagh G, Wambier CG. Rational hand hygiene during the coronavirus 2019 (COVID-19) pandemic. J American Acad Dermatol 2020; 82: e211. https://doi.org/10.1016/j. jaad.2020.03.090 